\title{
La polifuncionalidad de los marcadores discursivos en E/LE según unidad y posición*
}

\author{
ADRIÀ PARDO LliBRER \\ Universitat de València \\ Adria.Pardo@uv.es
}

\begin{abstract}
Resumen: Este trabajo aplica la segmentación en unidades discursivas del modelo Val.Es.Co. a los marcadores bueno, pues y hombre. Se analizan, así, las funciones descritas para estos marcadores en el Diccionario de partículas discursivas del español, las cuales se organizan según una relación de posición y ámbito con respecto a una unidad del discurso dada. Ello facilita una comparación de funciones entre estas formas e invita a diferentes desarrollos didácticos en el aula de E/LE según un usuario u otro.
\end{abstract}

Palabras clave: bueno, pues, hombre, polifuncionalidad, modelo Val.Es.Co.

\section{On teaching polyfunctional discourse markers from a discourse segmentation perspective in units and positions}

Abstract: Following the Val.Es.Co. model for discourse segmentation, this work analyzes the Spanish discourse markers bueno, pues and hombre in terms of discourse units and positions. This analysis allows to systematize the several meanings involved by these polyfunctional discourse markers and leads to some teaching applications.

Key words: bueno, pues, hombre, polyfunctionality, Val.Es.Co. model.

\section{Introducción}

Este trabajo teoriza sobre la cuestión de la polifuncionalidad de los marcadores del discurso (en adelante, MD) bueno, pues y hombre con visos a su enseñanza en E/LE. Estas formas presentan, junto con sus funciones nucleares, otras funciones secundarias, lo que hace de ellos un objeto de estudio difícil de organizar desde una orientación onomasiológica (al menos, en su aplicación docente). Nuestra propuesta, ya esbozada en un trabajo anterior (Pardo Llibrer 2019), aspira a una sistematización de las diferentes funciones implicadas en estos MD: a partir de su tratamiento en el Diccionario de partículas discursivas del español (Briz, Pons y Portolés 2008; en adelante, DPDE), se procede con un análisis de bueno, pues y hombre en términos de unidades discursivas y posiciones. En primer lugar $(\S 2)$, presentamos las tres variables contempladas en (la definición de) los MD polifuncionales ( $\S 2.1$ ), con las que revisar la descripción ofrecida en el DPDE (§ 2.2). A continuación (§ 3), aplicamos el sistema Val.Es.Co. de unidades discursivas a los ejemplos del $D P D E(\$ 3.1)$ : a partir de una relación según unidad y posición, puede compararse el lugar de bueno, pues y hombre en el discurso

*Este artículo ha sido posible gracias al proyecto de investigación FFI2016-77842-P, Unidades discursivas para una descripción sistemática de los marcadores del discurso en español (UDEMADIS), financiado por el MINECO, la AEI y los fondos FEDER. 
según las funciones que compartan ( $§ 3.2)$. Por último $(\S 4)$, se introduce la utilidad didáctica de esta metodología ( $§ 5)$. Unas conclusiones cierran esta disertación $(\S 6)$.

\section{Bueno, pues y hombre: marcadores polifuncionales}

Los MD constituyen una clase funcional. Esto es, las categorías utilizadas en su descripción atienden a los diferentes usos que pueden tener en el habla (Borreguero 2015). Contrariamente, la enseñanza de estos elementos suele ser de orientación onomasiológica (Pons 2015): se enumera un listado de formas y se les atribuyen sendas funciones. Tal caracterización es la que se sigue en la gramática (Martín Zorraquino y Portolés 1999) y en la lexicografía en general ( $D P D E$, Santos 2003), mientras que la pragmática obliga a una orientación semasiológica (describir funciones discursivas desempeñadas por MD independientemente de su forma). Para esta caracterización semasiológica, planteamos una definición operativa de los MD polifuncionales con base en tres variables $(\S 2.1)$, identificables de manera arquetípica en la entrada del DPDE para bueno, pero también replicables en la descripción de pues y hombre (§ 2.2).

\subsection{La polifuncionalidad de los marcadores del discurso: tres variables}

De entre las muchas definiciones de MD (vid. Schiffrin 1987, Fraser 1999, Portolés 1999, entre otros), asumimos la siguiente para los objetivos de este trabajo:

[E]xpresiones polifuncionales [...] permiten a los interlocutores identificar cuál es la orientación argumentativa de un discurso, cuánta información cabe inferir de un enunciado, cuál es la actitud del hablante hacia dicho enunciado o cómo se produce en la interacción (Salameh 2019: 54).

Al funcionar en el nivel pragmático, distinguimos (Pardo Llibrer 2018) los MD de otros elementos lingüísticos por su naturaleza suprasintáctica (no vienen exigidos por su combinación con otros sintagmas) y extraproposicional (pueden abstraerse de la oración en que aparecen sin repercutir en su significado). Esto implica que la función de un MD viene dada por el tipo de información procedimental que codifica (Sperber y Wilson 1993). Así, los usos de un MD pueden agruparse en tres funciones macro principales:

\begin{tabular}{|c|c|c|}
\hline \multicolumn{3}{|c|}{ TRES FUNCIONES MACRO } \\
(no-ideacionales, Halliday 1970; procedimentales, Sperber y Wilson 1993; adyacentes, Grupo Val.Es.Co. 2014) \\
\hline FUNCIÓN(ES) TEXTUAL(ES) & FUNCIÓN(ES) MODAL(ES) & $\begin{array}{c}\text { FUNCIÓN(ES) } \\
\text { INTERPERSONAL(ES) }\end{array}$ \\
\hline $\begin{array}{c}\text { Contribuyen a la } \\
\text { organización del discurso }\end{array}$ & $\begin{array}{c}\text { Reflejan una cierta actitud del } \\
\text { hablante con respecto a lo dicho }\end{array}$ & $\begin{array}{c}\text { Contribuyen a la interpelación de } \\
\text { los interlocutores }\end{array}$ \\
\hline $\begin{array}{c}\text { cohesión, ilación, } \\
\text { argumentación, } \\
\text { (re)formulación... }\end{array}$ & $\begin{array}{c}\text { atenuación, mitigación, } \\
\text { intensificación, evidencialidad, } \\
\text { epistemicidad... }\end{array}$ & $\begin{array}{c}\text { vocativos, acuerdo, } \\
\text { mantenimiento/cesión del turno, } \\
\text { control de contacto... }\end{array}$ \\
\hline
\end{tabular}

Figura 1. Macrofunciones de los marcadores del discurso 
Estas tres funciones simplifican la descripción (Halliday 1970, Grupo Val.Es.Co. 2014), pero también permiten reducir a tres categorías de análisis las funciones derivadas.

\subsection{Lo polifuncional en el Diccionario de partículas discursivas del español}

La cuestión de la polifuncionalidad de los MD se ve reflejada en el DPDE: las diferentes entradas ${ }^{1}$ recogen diferentes usos dentro de una misma forma, los cuales se ajustan -a grandes rasgos- a las funciones arriba expuestas. El caso de bueno, con tres entradas funcionalmente opuestas, es paradigmático. Así, bueno ${ }^{l}$ hace acopio de usos principalmente textuales (1), mientras que en bueno ${ }^{2}$ predominan los usos modales (2):

(1) A: ¿cómo sabías la dirección?

B: yo sabía que era esta la dirección// bueno pues por lo que me acuerdo de cuando estuve

(2) L: pero en los grandes almacenes síi que suelen haber

B: bueno hay / pero de esos de natación

En (1), bueno contribuye a la progresión temática del discurso (presenta una función continuativa o de ilación), mientras que en (2) sirve de anticipo a una matización que el hablante mitiga ('sí hay, pero'). Otros usos de bueno ${ }^{2}$ tienen función interpersonal:

(3) [Dos vecinas hablan sobre la conveniencia de instalar un ascensor]

A: los de-ahora le han dao permiso/ ahora lo han puesto

M: ¡Ay! ¿vees?

A: $§$ bueno $\S$

M: entonces $\downarrow$

A: PERO// a nosotros no nos interesa

En este caso, A se vale de bueno para validar la conformidad con lo dicho por M, si bien de manera parcial. Un uso que no solo se da en posición independiente, sino también de manera fático-apelativa al inicio de una intervención (en estilo directo) del interlocutor:

(4) A: sí sí sí/// el díaa/ ee-// eel día ese que avisaron de que habían entrao aquí $\uparrow / /$ estabaa/ estaba

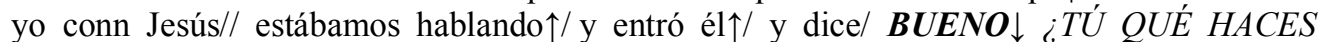
AQUÍ/ eh? (RISAS) a ver si vienes menos jeh?/ que él es un cural y digo y a mí qué me importa $\uparrow$ que sea cural yo me meto a monja $\downarrow$ si quiere

Estos ejemplos dan cuenta de cómo las funciones de bueno están sujetas a sus contextos de aparición. Esta situación se da también con vale, interpersonal en (5) y modal en (6):

(5) B: Te toca

C: Bueno $<$ vacilación $>$ cambiamos de tema si queréis

B: Vale $<$ risas $>$

(6) A: NO QUIERO QUE LO DEJEMOS/ ESO SÍ QUE LO TENGO CLARO§

B: §vale $\downarrow$ ahora dime cómo piensas aclararte/ ¿estando así?

Asimismo, el MD pues, bien con usos textuales tanto ilativos (7) como de inicio de tópico (8), bien con usos modales atenuantes (9), es igualmente polifuncional:

(7) - ¿Qué impresión le daba?

- Pues me sentía con mucha ilusión

(8) Pues venía a pedirle un favor

(9) Entonces, yo es que entiendo que en sí misma la marihuana pues es una trampa

\footnotetext{
${ }^{1}$ Todas las referencias no explícitas de bueno (Pons 2008), pues (Porroche 2008) y vale (Padilla 2008) remiten a sus respectivas entradas en el $D P D E$ (en línea: <www.dpde.es>).
} 
A colación de pues, sugeríamos en un trabajo anterior (Pardo Llibrer, 2019) cómo las funciones de esta forma pueden sistematizarse según su posición estructural en la conversación coloquial. Este acercamiento, sin embargo, parece limitado; no supera la perspectiva onomasiológica, ya que no contempla otros MD:

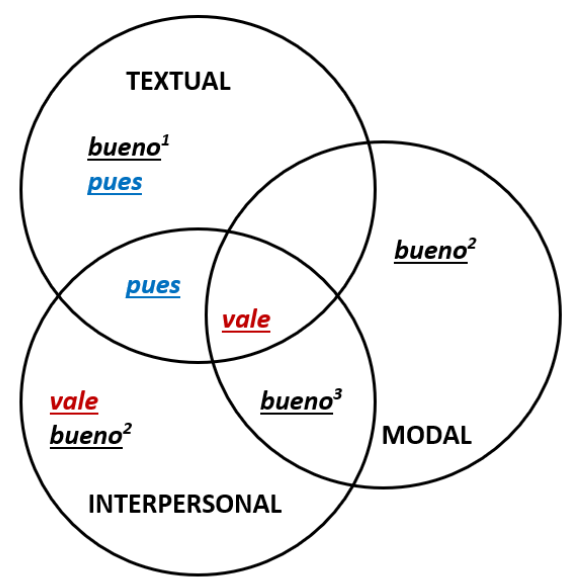

Figura 2. Totum revolutum de formas y funciones

Por ello, se procede a continuación con el análisis (ya seguido para pues) de bueno y vale en términos de unidades discursivas ( $\$ 3.1$ ), comparando la posición estructural de estos MD en el habla con las diversas funciones que puedan compartir (§ 3.2).

\section{Polifuncionalidad, unidades discursivas y posición}

Nuestra orientación es semasiológica: busca cómo organizar los MD según tres macrofunciones (Figura 1), sin atender a clasificaciones morfológicas. De este modo, el modelo Val.Es.Co. de unidades discursivas y posiciones $(\S 3.1)$ ofrece una visualización del ámbito predominante de bueno, pues y vale en función de si estas formas presentan una naturaleza (predominantemente) textual, modal o interpersonal (§ 3.2).

\subsection{El sistema Val.Es.Co. para la segmentación del discurso}

El modelo Val.Es.Co. (Grupo Val.Es.Co. 2014, Hernández 2018) plantea un sistema de ocho unidades (discurso, diálogo, intercambio, alternancia de turnos, turno, intervención, acto, subacto) para la sintaxis del habla. Estas unidades se organizan según dos niveles (monologal/dialogal) y tres órdenes (estructural/social/informativa): 


\begin{tabular}{|l|l|l|l|}
\hline NIVEL & \multicolumn{2}{|c|}{ ÓRDENES } \\
\hline Dialogal & ESTRUCTURAL & SOCIAL & INFORMATIVA \\
& $\begin{array}{l}\text { discurso } \\
\text { diálogo } \\
\end{array}$ & & \\
& intercambio & alternancia de turnos & \\
\hline Monologal & $\begin{array}{l}\text { intervención } \\
\text { acto }\end{array}$ & Turno & subacto \\
\hline
\end{tabular}

Figura 3. Unidades del sistema Val.Es.Co.

La unidad dialogal superior es un discurso dado, el cual integra diferentes diálogos resultantes de diferentes intercambios comunicativos. El orden social incumbe al turno y su alternancia, entendidos como la intervención de un hablante reconocida por su(s) interlocutor(es). El nivel monologal, por su parte, incluye la intervención y su constituyente inmediato, el acto, entendido como un enunciado divisible en subactos.

Especialmente relevante para nuestro estudio es esta última unidad. El subacto es la unidad monologal informativa mínima y se clasifica en subactos sustantivos (SS) o adyacentes (SA). Los SS tienen contenido conceptual y pueden ser directores (SSD) o subordinados (SSS), según si los segundos se supeditan a los primeros. Los SA, por contra, son elementos procedimentales que se corresponden con las tres funciones arriba expuestas: subactos adyacentes textuales (SAT), modales (SAM) o interpersonales (SAI). Así pues, los MD funcionan como subactos adyacentes, pero su caracterización con una u otra función está vinculada significativamente con su ámbito sobre unidades mayores (SSD, acto, intervención) y la posición con respecto a estas (posición inicial, inicial relativa -esto es, antecedida de otro $\mathrm{SA}-$, medial o final). Veamos su aplicación.

\subsection{El lugar en la conversación de bueno, pues y vale}

Siguiendo el modelo Val.Es.Co., podemos analizar los MD bueno, pues y vale según tres parámetros: función, ámbito sobre una unidad discursiva y posición con respecto a dicha unidad. De entrada, se reconocen dos funciones textuales en bueno:

(10) A: ¿cómo sabías la dirección?

B: \#yo sabía que era esta la dirección//\#\#\{SAT bueno SAT $\}\{$ SAT pues SAT $\}$ \{SSD por lo que me acuerdo de cuando estuve. SSD $\} \#$

(11) S: \#\{sss ¡aah!/ yo tengo una amiga que se ha montao una pi(s)cina pequeñita $\uparrow$ / que's para los bebés $\uparrow$ / perooo/ a(ho)ra/ aparte de'so se han apuntao/personas mayores///sss\} $\{$ SAT bueno/ SAT\} \{SSD pequeña/ diecisiete metros de pi(s)cina SSD $\} \#$

En (10), bueno tiene una función ilativa: se ubica en posición inicial con ámbito sobre todo el SSD ('por lo que me acuerdo de cuando estuve') y continúa tras una pausa (//) la información del segmento previo ('yo sabía que era esta la dirección'). En (11), por su parte, el ámbito sobre SSD reformula la información del segmento anterior ('pequeña'). Por lo que hace a los usos modales, bueno, en tanto que SAM y en posición inicial, tiene ámbito sobre el conjunto del segmento con fuerza ilocutiva (esto es, el acto: [I,A]):

(12) L: pero en los grandes almacenes síi que suelen haber

B: \#\{SAM bueno SAM $\}$ \{SSS hay / SSS $\}$ \{SSD pero de esos de natación SSD $\} \#$ 
Esta posición inicial se mantiene en los usos de bueno como SAI, pues los usos fáticoapelativos extienden su ámbito sobre el conjunto de la intervención:

(13) [...] \#\{SAI $B U E N O \downarrow$ SAI $\{$ SSD ¿ $T U U$ QUÉ HACES AQUÍ/ SSD\} \{SAI eh? SAI\} \{SAM (RISAS) SAM \#\# \# ver si vienes menos ¿eh?/ que él es un cura/\#\# digo y a mí qué me importa $\uparrow$ que sea cural yo me meto a monja $\downarrow$ si quiere\#

Este ámbito de bueno, en tanto que SAI, se mantiene con función de acuerdo parcial cuando este MD constituye por sí mismo una intervención independiente:

(14) [Dos vecinas hablan sobre la conveniencia de instalar un ascensor]

A: los de-ahora le han dao permiso/ ahora lo han puesto

M: ¡Ay! ¿vees?§

A: \#\{SAI $§$ bueno $\S$ SAI $\} \#$

M: entonces $\downarrow$

A: PERO// a nosotros no nos interesa

Si cada uno de estos usos se distribuye según una relación cruzada de unidad-posición, se obtiene (lo que viene a llamarse) una parrilla con las principales funciones:

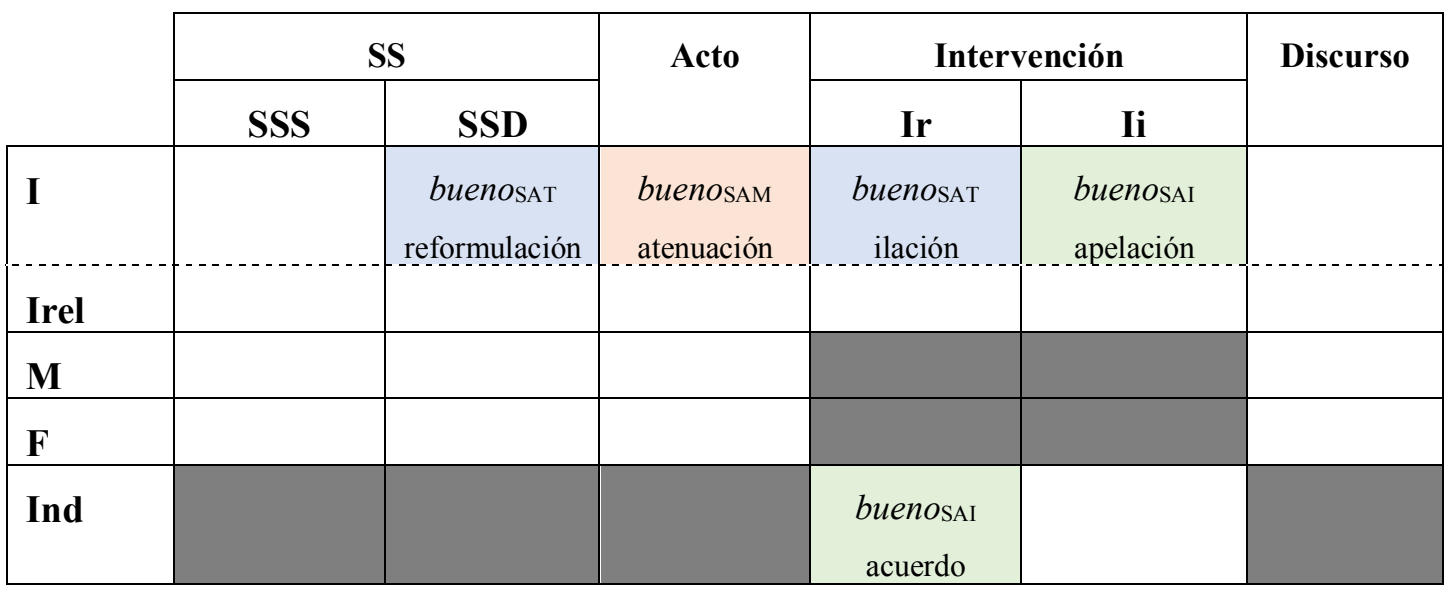

Figura 4. Relación unidades-posición-función en bueno

Las funciones de bueno pueden simplificarse en una visualización gráfica que facilita su descripción metalingüística. Del mismo modo, es posible incorporar a esta parrilla las funciones de otros $\mathrm{MD}$, a fin de observar distribuciones complementarias $\mathrm{y} / \mathrm{o}$ coincidencias de uso. Así, encontramos dos funciones principales en vale:

(15) A: NO QUIERO QUE LO DEJEMOS/ ESO SÍ QUE LO TENGO CLARO§

B: \#\{SAM $§ \mathbf{v a l e} \downarrow$ SAM $\{$ SSD ahora dime cómo piensas aclararte/ ¿estando así? SSD\}\#

(16) B: Te toca

C: Bueno $<$ vacilación $>$ cambiamos de tema si queréis

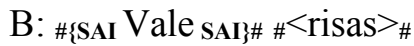

Con el primer vale (15), A atenúa una contrargumentación ('cómo piensas aclararte') con respecto a lo dicho por B ('eso lo tengo claro'); mientras que, en (16), la función de SAI en posición independiente valida la intervención anterior, como bueno en (14). Así: 


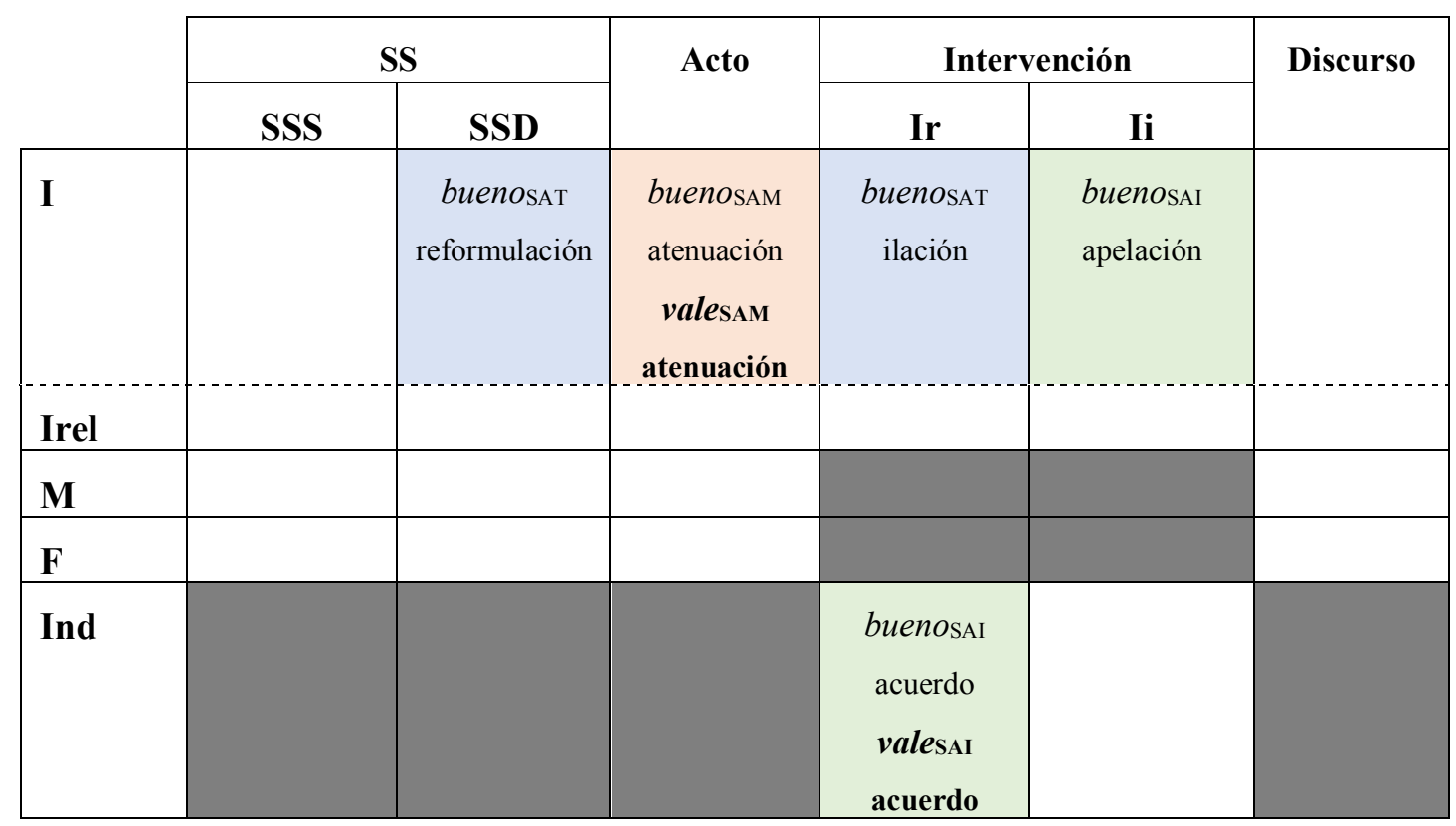

Figura 5. Relación unidades-posición-función en bueno y vale

Como vemos, la polifuncionalidad de vale no es tan amplia como la de bueno. Mientras que este último presenta usos textuales (principalmente en posición inicial), vale vislumbra una preferencia por la función interpersonal en posición inicial, si bien puede coincidir como modalizador con bueno. Así las cosas, es posible incorporar a esta parrilla las funciones de pues (ya descritas en una primera aplicación -Pardo Llibrer 2019- de esta metodología; vid. §2.2). El resultado es el siguiente:

\begin{tabular}{|c|c|c|c|c|c|c|}
\hline & \multicolumn{2}{|c|}{ SS } & \multirow[t]{2}{*}{ Acto } & \multicolumn{2}{|c|}{ Intervención } & \multirow[t]{2}{*}{ Discurso } \\
\hline & SSS & SSD & & Ir & Ii & \\
\hline I & & $\begin{array}{c}\text { buenoSAT } \\
\text { reformulación } \\
\text { puesSAT } \\
\text { ilación }\end{array}$ & $\begin{array}{c}\text { buenosAm } \\
\text { atenuación } \\
\text { valesAm } \\
\text { atenuación }\end{array}$ & $\begin{array}{c}\text { buenoSAT } \\
\text { ilación } \\
\text { puessAT } \\
\text { ilación }\end{array}$ & $\begin{array}{c}\text { buenoSAI } \\
\text { apelación } \\
\text { puessAT } \\
\text { inició tópico }\end{array}$ & \\
\hline Irel & & & $\begin{array}{c}\text { puessam } \\
\text { atenuación }\end{array}$ & & & \\
\hline $\mathbf{M}$ & & $\begin{array}{c}\text { puessaT } \\
\text { formulación }\end{array}$ & $\begin{array}{c}\text { puessam } \\
\text { atenuación }\end{array}$ & & & \\
\hline $\mathbf{F}$ & & & & & & \\
\hline Ind & & & & $\begin{array}{c}\text { buenosAI } \\
\text { acuerdo } \\
\text { valesAI } \\
\text { acuerdo }\end{array}$ & & \\
\hline
\end{tabular}

Figura 6. Relación unidades-posición-función en bueno, vale y pues

De acuerdo con esto, pues presenta un alto grado de coincidencia con bueno tanto en sus funciones textuales como en sus funciones modales, si bien sus usos formulativo (esto 
es, SAT en [M, SSD]) y atenuante (SAM en [IREL, A]) tienden hacia posiciones medias. Por otra parte, es destacable cómo pues no presenta usos interpersonales prototípicos (no puede estar en posición independiente), pero sí coincide estructuralmente con otros MD en función de SAI (como bueno, en [I, Ii], con valor apelativo).

La utilidad de esta organización en parrillas de funciones según unidad y posición no se reduce a la mera teorización sobre la distribución de los $\mathrm{MD}$, sino que supone un primer paso para evitar ciertos reduccionismos de la metodología formalista. No se trata tanto de enumerar las diferentes funciones relativas a una forma (bueno ${ }^{1}$, bueno $^{2}$, etc.), sino más bien de perfilar tendencias en la correspondencia entre un ámbito de aparición y una macrofunción. Con estos datos (Figura 6), la ubicación de un MD hacia el inicio es indiciaria de una función textual, mientras que los MD interpersonales tienden constituir reacciones en sí mismos. Por último, lo modal tiene ámbito sobre lo ilocutivo (el acto), por lo cual su posición está menos especializada estructuralmente.

La confluencia de parrillas que aquí se propone exigiría, para su aplicación, un dominio del metalenguaje (conocimientos de pragmática teórica, análisis conversacional y familiarización con la segmentación en unidades discursivas) que no tienen por qué conocer sus potenciales usuarios. Por ello, planteamos a continuación algunas posibilidades (meta)didácticas de esta segmentación según unidad-posición-función para el aula de E/LE, simplificando la parrilla de tal modo que devenga en categorías más operativas para los docentes y en nociones más intuitivas para los estudiantes.

\section{Acerca de algunas posibilidades didácticas de las parrillas de bueno, pues y vale}

Una vez identificado el ámbito más recurrente de los tres MD (según sean SAT, SAM o $\mathrm{SAI}$ ), caben tres consideraciones previas para una posible aplicación didáctica:

a. En primer lugar, es necesario traducir conceptual y terminológicamente las unidades del modelo Val.Es.Co. en nociones más comunes en el aula de E/LE.

b. En segundo lugar, conviene colapsar las categorías relativas a la posición, para hacer más accesibles los conceptos de posición inicial relativa o independiente.

c. En tercer lugar, hay que ofrecer al docente un equivalente práctico de las tres macrofunciones en que la descripción se centra (inasumibles por el alumno).

Teniendo esto en cuenta, cabe la posibilidad de ofrecer al profesor de E/LE (como primer usuario inmediato) una organización de los MD en términos más gramaticales: las unidades discursivas más cercanas a la sintaxis (SSS/SSD) pueden corresponderse con las nociones de cláusula y oración respectivamente, mientras que el acto se acerca a la idea de enunciado (oración cargada comunicativamente) y la intervención (por su naturaleza dialogal) puede subsumirse en la idea de turno (enunciado que responde). Si, aparte, reducimos a una las posiciones iniciales, la distribución de MD es la que sigue: 


\begin{tabular}{|c|c|c|c|c|}
\hline & Cláusula & Oración & Enunciado & Turno \\
\hline INIClO & $\begin{array}{l}\text { buenoreformulación } \\
\text { puesilación }\end{array}$ & $\begin{array}{l}\text { bueno ilación } \\
\text { puesilación }\end{array}$ & $\begin{array}{l}\text { bueno }_{\text {ilación }} \\
\text { vale }_{\text {atenuación }}\end{array}$ & $\begin{array}{l}\text { bueno apelativo } \\
\text { puesinicio tópico }\end{array}$ \\
\hline MITAD & & pues formulación & pues atenuación & \\
\hline FINAL & & & & \\
\hline INDEPENDIENTE & & & & $\begin{array}{l}\text { bueno acuerdo } \\
\text { vale }_{\text {acuerdo }}\end{array}$ \\
\hline
\end{tabular}

Figura 7. Adaptación de la relación unidades-posición-función en bueno, vale y pues

Esta distribución presenta dos cambios básicos con respecto a la Figura 6: por un lado, la forma de los MD descritos es independiente de la función desempeñada (como muestra la correspondencia cromática con las tres macrofunciones); por otro lado, hace evidentes algunas excepciones en la preferencia posicional que dificultan su aplicación didáctica. En este sentido, la función formulativa de pues comparte ámbito (medial) con su función modal; mientras que otros usos modales, como el de vale, se ubican al inicio. En cuanto a lo interpersonal, bueno presenta usos (apelativos) no independientes.

Estas excepciones implican un problema teórico en el estudio de los MD, pero no tienen por qué serlo en su aplicación a la enseñanza. Por ello, una primera solución práctica podría ser (en línea con las tres consideraciones previas expuestas más arriba) la siguiente:

\begin{tabular}{|c|c|c|c|c|}
\hline & Cláusula & Oración & Enunciado & Turno \\
\hline INICIO & $\begin{array}{l}\text { bueno reformulación } \\
\text { puesilación }\end{array}$ & $\begin{array}{l}\text { bueno ilación } \\
\text { puesilación }\end{array}$ & bueno $_{\text {ilación }}$ & $\begin{array}{l}\text { bueno apelativo } \\
\text { puesinicio tópico }\end{array}$ \\
\hline MITAD & & pues formulación & $\begin{array}{l}\text { puesatenuación } \\
\text { vale atenuación }\end{array}$ & \\
\hline FINAL & & & & \\
\hline INDEPENDIENTE & & & & $\begin{array}{l}\text { bueno acuerdo } \\
\text { vale }_{\text {acuerdo }}\end{array}$ \\
\hline
\end{tabular}

Figura 8. (Re)adaptación de la relación unidades-posición-función en bueno, vale y pues

Los criterios para esta modificación son los siguientes: la función modal en vale y pues se sitúa a menudo en una posición inicial relativa, entendida (por defecto) como medial por el usuario no especializado. Por su parte, el bueno apelativo es un SAI en [I, Ii] pero, al ser una intervención iniciativa, la apelación se entiende como una estructura marco para introducir una nueva información (por lo que no está tan lejos de adquirir un cierto valor textual subsidiario). Esta reformulación de la parrilla ofrece tres ámbitos claros para los SAT, SAM y SAI. Ello permite acercarse a bueno, pues y vale no tanto por las funciones que contengan sino por las funciones que (de entre las tres básicas) puedan desempeñar. Esta visualización se puede simplificar un punto para los alumnos: 


\begin{tabular}{|l|c|c|c|}
\cline { 2 - 4 } \multicolumn{1}{c|}{} & Parte de oración & Oración & Respuesta \\
\hline INICIO & bueno/pues & bueno/pues & bueno/pues \\
\hline MEDIO & & bueno/pues/vale & \\
\hline FINAL & & & \\
\hline SOLO & & & bueno/vale \\
\hline
\end{tabular}

Figura 9. Posible adaptación para los alumnos

En este punto, la Figura 9 reflejaría el grado máximo de simplificación de la distribución de bueno, pues y vale de acuerdo con el análisis inicial de la parrilla según unidades y posición. Ahora bien, tres problemas persisten en esta aplicación didáctica:

a. Primero, la función formulativa (SAT) de pues queda subsumida por la función atenuadora (SAM), dada su coincidencia posicional (posición media). Por ello, se necesita un marbete para la posición media que comprenda ambas funciones.

b. Segundo, la operatividad para el alumno de las tres macrofunciones es bastante cuestionable: la idea de lo textual en los MD puede explicitarse por su relación con los conectores, pero la separación entre lo modal y lo interpersonal (además de solaparse) resulta menos objetivable.

c. Tercero, aunque una explicación en términos de posición sea la más accesible para un alumno, es igualmente conveniente relacionarla con un concepto no tan metalingüístico como es el concepto de ámbito.

Pensamos que una solución operativa a estos tres problemas puede ser la de transformar la parrilla de la Figura 9 en una relación de términos más intuitivos:

\begin{tabular}{|l|c|c|c|}
\cline { 2 - 4 } \multicolumn{1}{c|}{} & Parte de oración & Oración & Respuesta \\
\hline INICIO & seguir hablando & seguir hablando & $\begin{array}{c}\text { seguir hablando } \\
\text { empezar a hablar }\end{array}$ \\
\hline MEDIO & & pensar qué decir & \\
\hline FINAL & & & \\
\hline SOLO & & & seguir escuchando \\
\hline
\end{tabular}

Figura 10. Adaptación intuitiva para los alumnos

Estas nociones se justifican, para los fines de este trabajo, en la medida en que solventan los problemas antes enunciados. En primer lugar, tanto la función formulativa (concerniente a la planificación del habla sobre la marcha) como la atenuación (concebida como una modalización de actos de habla potencialmente polémicos) enlazan con el acto de pensar el decir; de ahí la etiqueta común de pensar qué decir (lo textual vendría a pensar más bien el dictum y lo modal, el modus; García Negroni y Tordesillas 2001). En segundo lugar, y en línea con esto, lo textual se relaciona con el hablar mismo (funciones ilativas -seguir hablando-y de inicio-empezar a hablar- de tópico) y lo interpersonal (funciones fáticas y de validación), con la escucha activa (seguir escuchando). Finalmente, estas nociones suponen una alternativa (más o menos 
intuitiva) al concepto (muy metalingüístico) de ámbito para las funciones de bueno, pues y vale. Del mismo modo que los MD varían según estén al inicio, en el medio o solos; así también su función entronca con el hablar, el pensar o el escuchar:

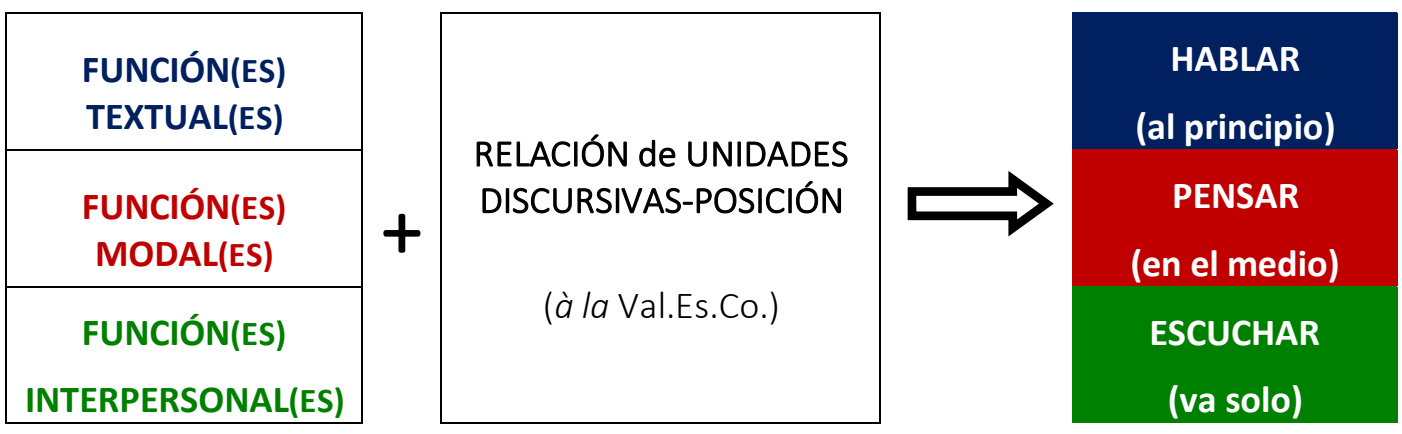

Figura 11. El hablar, el pensar y el escuchar según su posición estructural

El hablar, el pensar y el escuchar se entienden aquí como una metáfora de las tres macrofunciones textual, modal e interpersonal respectivamente. El análisis de MD desde un enfoque onomasiológico (enumeración de los usos de cada elemento) deviene en un totum revolutum de formas y funciones sin mayor concierto. La aplicación del modelo Val.Es.Co. para la segmentación en unidades discursivas y posiciones sirve de herramienta con que delimitar los ámbitos de actuación de bueno, pues y vale según estos sean SAM, SAT o SAI indistintamente. La traducción de lo textual como aquello que se utiliza para el hablar (la elaboración de la información en el discurso), lo modal para el pensar (porque se piensa qué decir y cómo) y lo interpersonal para el escuchar (papel más reactivo) obvia las unidades simplificadas (Figura 10) de cláusula, oración y respuesta; al tiempo que generaliza, en tres posiciones muy básicas, esta tríada de usos.

\section{Conclusiones}

La enseñanza de MD en el aula de E/LE suele seguir un enfoque onomasiológico acrítico. Es decir, se disponen listados de formas con una o más funciones. El punto de vista semasológico, no obstante, parte de tres macrofunciones fácilmente identificables que pueden asociarse a tres ámbitos generales: lo textual, en posiciones iniciales; lo modal-formulativo, en el medio; y lo interpersonal, independiente. Esta correspondencia entre posición y función es posible gracias a la aplicación del sistema Val.Es.Co. de segmentación en unidades discursivas, punto de partida para simplificar paulatinamente la parrilla hasta llegar a una explicación en términos del hablar, el pensar y escuchar.

\section{Bibliografía}

BorREgUERO, M. N. (2015). «A vueltas con los marcadores del discurso: de nuevo sobre su delimitación y sus funciones». En A. Ferrari, L. Lala (ed.), Testualità, Fondamenti, Unità, Relazioni (pp. 151-170). Florencia: Franco Cesati.

BRIZ, A., Pons, S. y Portolés, J.(2008). Diccionario de partículas discursivas del español. En línea: $<$ http://dpde.es $>$

FRASER, B. (1999). «What are discourse markers?», Journal of Pragmatics, 31, pp. 931-952. 
García Negroni, M. ${ }^{a}$ M.y M. Tordesillas (2001). La enunciación en la lengua. De la deixis a la polifonía. Madrid: Gredos.

GRUPO VAL.Es.Co. (2014). «Las unidades del discurso oral. La propuesta Val.Es.Co. de segmentación de la conversación (coloquial)», Estudios de Lingüística del Español, 35, pp. 1373.

HALliDAY, M. y A. KIRKWOOD (1970). «Language structure and language function». En J. Lyons (ed.), New horizons in linguistics (pp. 140-165). Harmondsworth: Penguin.

HERNÁNDEZ RUIZ, A. (2018). «Hacia una sistematización del acto: problemas en su segmentación. Los actos sin SSD». En M. Díaz, J. Diz, A. Pérez, A. Varela (dirs.), Novas perspectivas na lingüistica aplicada (pp. 185-196). Lugo: Axac.

MARTín ZORRAQUinO, M. ${ }^{a}$ A. y PORTOLÉS, J. (1999). «Los marcadores del discurso». En I. Bosque \& V. Demonte (dirs.), Gramática descriptiva de la lengua española (pp. 4051-4213). Madrid: Espasa Calpe, vol.3.

Padilla, X. (2008). «Vale». En Briz, A., Pons, S. \& Portolés, J. (dirs), Diccionario de partículas discursivas del español. En www.dpde.es

PARDO LliBRER, A. (2019). «La enseñanza de los marcadores del discurso en el aula de E/LE desde la segmentación en unidades de la sintaxis del habla: el caso de "pues"», Revista Foro de profesores de E/LE, 15, pp. 179-190.

POnS, S. (2008). «Bueno». En Briz, A., Pons, S. \& Portolés, J. (dirs), Diccionario de partículas discursivas del español. En www.dpde.es

Pons, S. (2015). La enseñanza de la pragmática en el aula de E/LE. Madrid: Arco/Libros.

Porroche, M. (2008). «Pues», en Briz, A., Pons, S. \& Portolés, J. (dirs), Diccionario de partículas discursivas del español. En www.dpde.es

PORTOLÉs, J. (1998). Marcadores del discurso, Barcelona, Ariel.

SALAMEH, S. (2019). Reformulation and neighboring categories: a theoretical-experimental approach through the Spanish discourse marker 'o sea'. Tesis Doctoral, Universitat de València / Ruprecht-Karls-Universität Heidelberg.

SANTOS Río, L. (2003). Diccionario de partículas, Salmanca: Luso-Española de ediciones.

SCHIFFRIN, D. (1987). Discourse markers. Cambridge: Cambridge University Press.

WiLSON, Dy Dan SPERBER (1993). «Linguistic form and relevance». Lingua 90 (1-2), pp. 1-25. 